\title{
Accident Management System Based on Vehicular Network for an Intelligent Transportation System in Urban Environments
}

\author{
Yusor Rafid Bahar Al-Mayouf $\left(\mathbb{D},{ }^{1}\right.$ Omar Adil Mahdi $\left(\mathbb{D},{ }^{1}\right.$ Namar A. Taha, \\ Nor Fadzilah Abdullah, ${ }^{2}$ Suleman Khan $\mathbb{1}^{3},{ }^{3}$ and Muhammad Alam ${ }^{4}$ \\ ${ }^{1}$ Department of Computer Science, College of Education for Pure Science (Ibn Al-Haitham), University of Baghdad, Iraq \\ ${ }^{2}$ Centre of Advanced Electronic and Communication Engineering, Faculty of Engineering and Built Environment, \\ Universiti Kebangsaan Malaysia, Malaysia \\ ${ }^{3}$ School of Information Technology, Monash University, Bandar Sunway 47500, Malaysia \\ ${ }^{4}$ Department of Computer Science and Software Engineering, Xian Jiaotong-Liverpool University, Suzhou, China
}

Correspondence should be addressed to Yusor Rafid Bahar Al-Mayouf; engineer_mu2007@yahoo.com and Suleman Khan; suleman.khan@monash.edu

Received 23 February 2018; Accepted 8 April 2018; Published 27 May 2018

Academic Editor: Syed Hassan Ahmed

Copyright (c) 2018 Yusor Rafid Bahar Al-Mayouf et al. This is an open access article distributed under the Creative Commons Attribution License, which permits unrestricted use, distribution, and reproduction in any medium, provided the original work is properly cited.

As cities across the world grow and the mobility of populations increases, there has also been a corresponding increase in the number of vehicles on roads. The result of this has been a proliferation of challenges for authorities with regard to road traffic management. A consequence of this has been congestion of traffic, more accidents, and pollution. Accidents are a still major cause of death, despite the development of sophisticated systems for traffic management and other technologies linked with vehicles. Hence, it is necessary that a common system for accident management is developed. For instance, traffic congestion in most urban areas can be alleviated by the real-time planning of routes. However, the designing of an efficient route planning algorithm to attain a globally optimal vehicle control is still a challenge that needs to be solved, especially when the unique preferences of drivers are considered. The aim of this paper is to establish an accident management system that makes use of vehicular ad hoc networks coupled with systems that employ cellular technology in public transport. This system ensures the possibility of real-time communication among vehicles, ambulances, hospitals, roadside units, and central servers. In addition, the accident management system is able to lessen the amount of time required to alert an ambulance that it is required at an accident scene by using a multihop optimal forwarding algorithm. Moreover, an optimal route planning algorithm (ORPA) is proposed in this system to improve the aggregate spatial use of a road network, at the same time bringing down the travel cost of operating a vehicle. This can reduce the incidence of vehicles being stuck on congested roads. Simulations are performed to evaluate ORPA, and the results are compared with existing algorithms. The evaluation results provided evidence that ORPA outperformed others in terms of average ambulance speed and travelling time. Finally, our system makes it easier for ambulance to quickly make their way through traffic congestion so that the chance of saving lives is increased.

\section{Introduction}

The concept of a smart city is linked to a paradigm shift where interest is being directed towards proposals and technological initiatives, which seek to make cities smart so that the quality of the lives of the people living in these cities can be improved $[1,2]$. For instance, in Europe, the European Commission has established the European Initiative on Smart Cities in 2010. This initiative seeks to address the four important elements in the life of a city: climate management systems, building, transport, and electricity.

One of the main elements that smart cities seek to contain is the issue of increasing levels of road accidents, which have resulted from increasing numbers of vehicles, leading to congestion. It has been proven that vehicle accidents are one of the main causes of fatalities with more than 100 deaths being reported in the United States on a daily basis [3]. The same report indicates that the year 2007 experienced 
more than 43,000 deaths as a result of over 10 million reported accidents. Moreover, in most of the countries in the world, a major cause of accidents is poor infrastructure for managing traffic. The concept of traffic management systems refers to intelligent public transport mechanisms founded on information obtained in real-time, which helps drivers to avoid congested areas and increase safety while also ensuring that vehicles operate in a manner that is more environmentally friendly. Within the last few years, researchers, both from academic institutions and from industry have taken advantage of the progress made in wireless sensing technology to make the current traffic management systems more effective and ensure their ability to cope with the issues within the smart city discussed above.

Traffic congestion, as one of the leading problems affecting modern societies, has been given increasing attention. The delay in the arrival of emergency services at accident scenes like ambulances, the police, and fire, and rescue operations is one of the critical results of traffic congestion. This is an undesirable situation as the safety of the general public depends on these services being able to arrive at the scene of an accident in the efficient and shortest time possible. Intelligent transportation systems (ITSs) have not yet been able to resolve the challenge of traffic congestion. For example, Google Maps still uses communication networks that exist such as Wi-Fi, global positioning system (GPS), and cellular networks to plan journeys and mitigate the challenge of being stuck in traffic. However, apart from being expensive, these technologies still do not have the capacity to respond with speed when there is an emergency resulting from a road accident. These systems have limited access to realtime traffic data. Hence, it is important that these emergency services should be equipped with a system that can enhance the adaptability of route planning so information can be collected more efficiently and real-time traffic data can be further exploited to avoid traffic congestion.

Firstly, emerging vehicular ad hoc networks (VANETs) can be used to deliver on ITS system with improved communications capabilities for obtaining real-time traffic information more efficiently and in a cost-effective manner [4]. VANETs support vehicle-to-roadside unit (V2R) and vehicle-to-vehicle communications $[5,6]$, so that real-time updates can be transmitted from and to vehicles and roadside units (RSUs) $[7,8]$. This real-time information which will be collected can then be consequently used for route planning in individual vehicles [9], freeway-traffic-flow management $[10,11]$, and vehicle localization [12-14]. Secondly, once the real-time traffic information has been obtained, various algorithms can be designed with the aim of discovering the most efficient routes that individual vehicles can take $[15,16]$. However, if the route planning is deployed in a manner which is not coordinated, this could lead to even more congestion. While most of the available routes planning global algorithms pay attention to the improvements linked to networks, they generally overlook driver preferences, such as travel distances and times. This is important, particularly if one considers that replanning decisions are a consequence of the need to avoid traffic congestion and balancing of traffic as opposed to the discovery of optimal routes. Hence, some drivers may actually prefer to take longer routes even though this may entail higher costs. Hence, the design of algorithms should be done in a way that considers both the reduction of the average cost of running the vehicle and network traffic.

To this end, we propose an accident management system based on VANET for ITS in urban environments. The major contributions of this paper are listed as follows:

(1) We provide an accident management system that makes use of VANETs coupled with systems that employ cellular technology in public transport. The provided system ensures the possibility of real-time communication among vehicles, ambulances, hospitals, roadside units, and central servers. A multihop optimal forwarding algorithm is used in this system, which provides the optimal route between the sources (accident vehicle) and the destination (ambulance). This can lower the amount of time required to alert an ambulance that it is required at an accident scene.

(2) We propose an optimal route planning algorithm which provides the optimal route algorithm from ambulance to accident vehicle and then to hospital. The proposed algorithm employs VANET communication proficiencies so that vehicles can mitigate the challenge of congestion. With the aim of balancing the preferences of the drivers and the need for overall smoothness in the traffic flow, we consider vehicle travel costs and network spatial utilization.

The remainder of this paper is organized as follows. Section 2 of this paper looks at related works on route planning, while Section 3 discusses the system model. Section 4 of the paper presents the traffic routing algorithms, while Section 5 delivers a demonstration of the performance evaluation. The paper is finally concluded in Section 6.

\section{Related Works}

There are many consequences of an unbalanced flow of traffic and the subsequent accidents and incidents it causes: arriving late and suffering added cost are some of the results drivers have to contend with. Route navigation and planning can, however, mitigate the challenges linked to traffic congestion.

Some of the methods which can be used to manage and plan vehicle routes include the shortest-route based GPS navigation, advance route reservation, and accident duration prediction [17-19]. However, all these methods do not have the capacity to deliver immediate information when an accident or congestion happens suddenly, considering the fact that they lack a timely update capacity to deliver information on traffic conditions. Hence, the availability of real-time traffic information becomes vital for supporting vehicular real-time route planning algorithms.

Loop directors and cellular systems are the most commonly used methods in standard ITS when collecting timevarying traffic condition data. The use of mobile sensors connected through cellular networks was investigated, for the collection of real-time traffic information for the forecasting 
of traffic or experimental research reconstruction [20-22]. A traffic management system which employs loop detectors for continuously measuring traffic and also monitoring of arterial routes is introduced in [15]. However, both loop detectors and cellular-based systems have their own challenges. With regard to cellular services, they are not designed for the purpose of collecting traffic data and the transmission of data through them can also be expensive. When too much data is transmitted through such networks, they may also crash due to added congestion. Loop detectors can also be quite expensive to deploy. Also, when it comes to short distance transmission, inaccurate position measurements can also present problems, especially in networks that are dense, resulting in the degrading of the performance of the route management planning [23].

VANETs V2R and V2V methods of communication have the capacity of making the delivery of real-time messages much quicker, more efficient, and cost-effective when compared to prevailing methods, even in shorter distances and networks that are thick [24, 25]. More importantly, the collection and distribution of data can be enhanced by the RSUs, in VANETs making it possible for coordinated route planning to be achieved for clusters of vehicles [26]. With the aim of improving the quality of experience, the multimedia delivery application can be supported by a vehicular network based on the multimedia system, which could still be a victim of extensive delays in transmission $[27,28]$.

A variety of studies have concentrated on real-time route planning assisted by VANETs. A method for distributed route planning was advanced to mitigate the challenge of congestion through the employment of real-time data obtained from VANETs, with the increase in the flow of traffic [15]. With regard to fuel saving in vehicles, [29] has designed a navigation system which assists drivers to steer away from congested roads. However, the lack of coordination and selfish behavior of drivers could lead to more congestion when the individualuser manual schemes are deployed. Hence, the requirement for the routes of groups of vehicles to be planned jointly with the aim of balancing the traffic network exists. Multivehicle route planning is considered in $[30,31]$. However, these works do not pay attention to the preferences of drivers, nor the average cost of travelling. It also remains unexplained how communications in VANETs can affect the route planning algorithm.

Hence, in this study, a route planning algorithm which is globally optimal is proposed to assist vehicles to avoid traffic congestion as a consequence of accidents within an urban setting. It is expected that the collected real-time traffic data and the efficient route decision-making will help facilitate better use of the resources within the road network, resulting in the average costs associated with travelling in a vehicle being reduced.

\section{System Model and Architecture}

In this section, we aim to provide an accident management system. We initially introduce the system model and then provide its architecture.
3.1. System Model. Within the urban environment, we consider a vehicular network that incorporates intersections and their roads. Within every road, there are two lanes in which the vehicles move in opposite directions as is illustrated in Figure 1.

The circle, in which intersection ID is found, represents the intersection, while the arrow is an indication of the direction in which the vehicles in the lane are travelling. Hospitals 1 and 2 denote the destinations that are assumed to be placed within two of the intersections. In each intersection, an RSU is located. There is one ambulance located near each hospital. All parts of the system are connected to the central server. When vehicles move through the road, they do so in either a similar or opposite direction. We make the assumption that all ambulances and vehicles have an on-board navigation mechanism and the capacity to use wireless communication similar to assumption in [32]. Within each ambulance and vehicle, there is a digital map that also contains knowledge of the locations of neighboring intersections. In addition, all vehicles are able to obtain necessary information regarding the location, speed, and direction of neighboring vehicles, which is maintained in tables accessible to neighbors by exchanging of periodic messages. While still generalizing, we also make the assumption that all vehicles have a range of transmission. We also make the assumption that the source node is armed with location services that allow it to obtain destination position in the event that it is needed $[33,34]$.

3.2. System Architecture. The accident management system consists of five units as is illustrated in Figure 2: vehicular, central server, RSUs, ambulance, and hospital. In this section, we will discuss each unit in detail as follows.

3.2.1. Vehicular Unit. The role of the vehicular unit is to manage the overall activities that the vehicle carries out, including the detection of accidents, while also maintaining communication with other units. In each vehicular unit, there are three modules.

(1) Sensor module: it is used to monitor and control different sensors attached to the vehicle. Our system uses two kinds of sensors for the purpose of detecting accidents: biomedical sensors which detect the individual's heart rate, temperature, and pressure and vehicle sensor which monitor acceleration, vibration, and the deployment of the airbag. Both sensors have their strengths and weaknesses. For instance, the vehicle sensor is easier to use but will not provide a precise picture of the accident. While it is able to detect that an accident has occurred, it is unable to give details regarding the gravity of the accident and how badly the passengers in the accident require medical attention. On the other hand, biomedical sensors are prone to raising false alarms. In order to mitigate against this challenge, we propose that both sensors should be used simultaneously. All readings provided by the sensors are always being measured and delivered to the local processor in the vehicle. The 


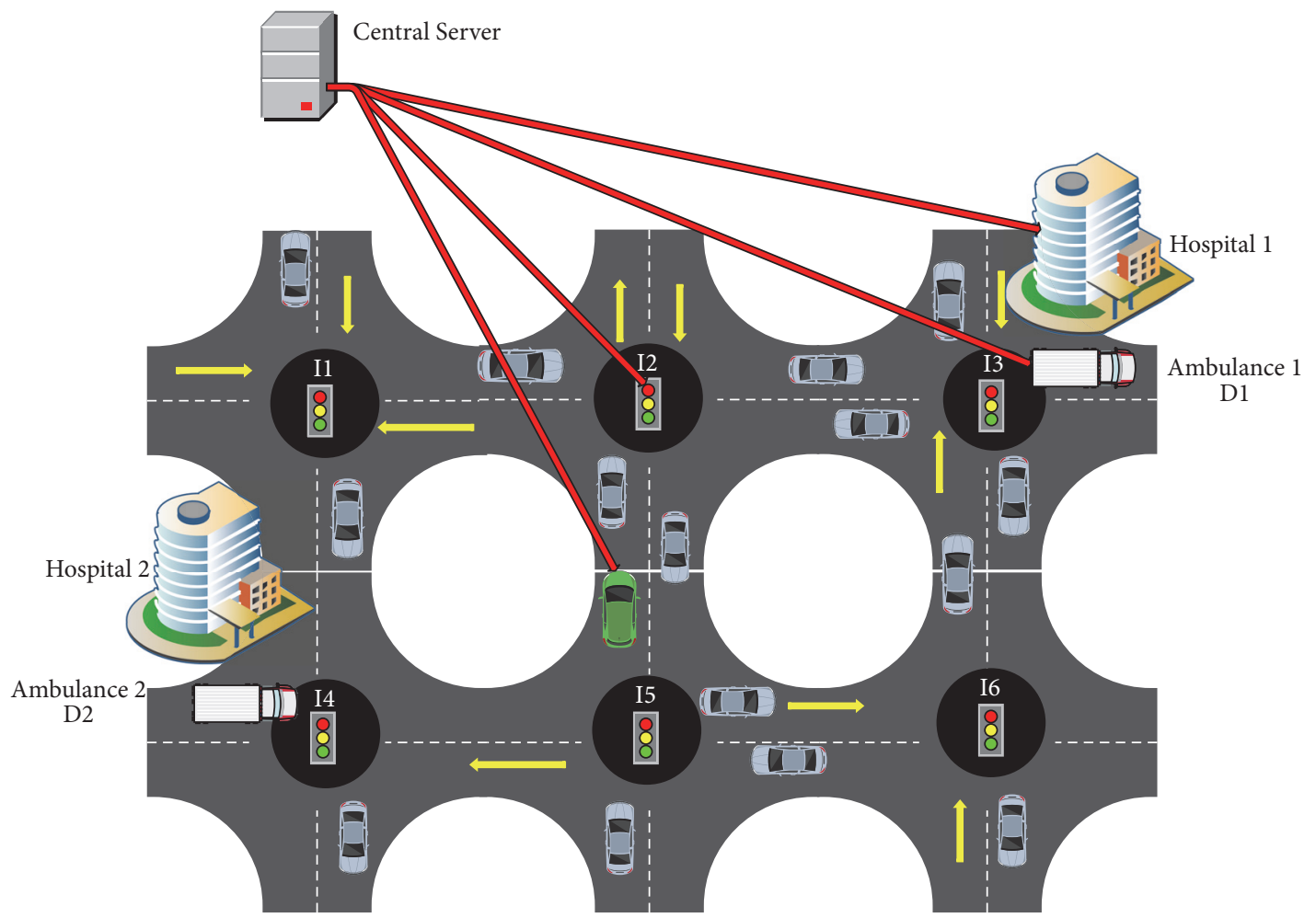

Figure 1: Model of accident management system.

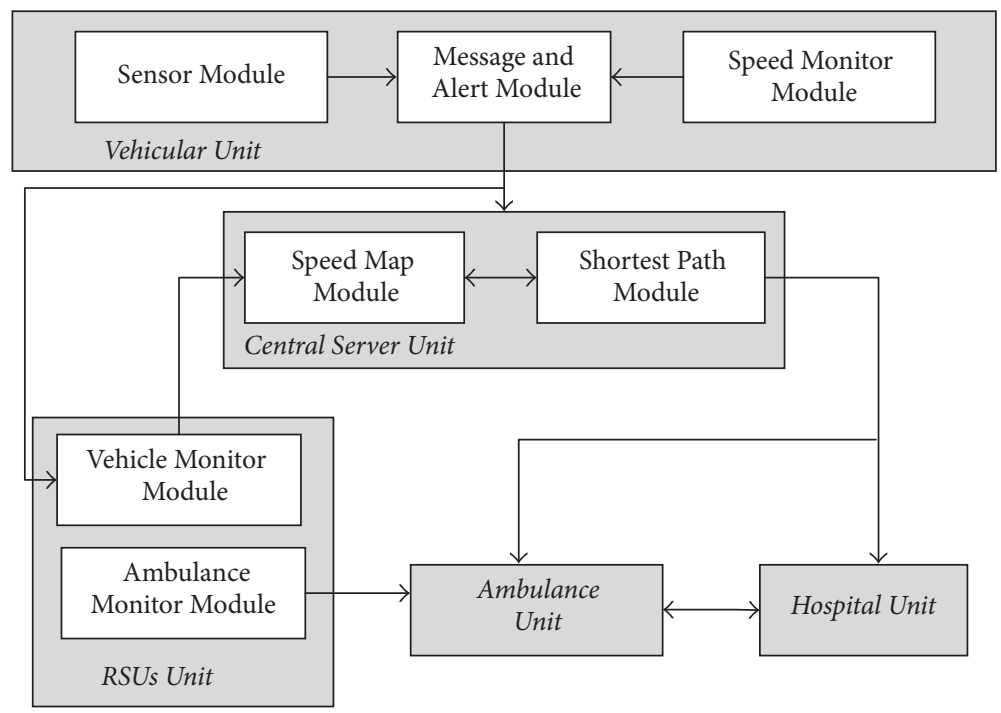

FIGURE 2: Architecture of accident management system.

processor is used for setting the threshold level for each reading of the sensor. The role of the processor is to continuously check the reading of the sensor against the threshold value. Once any abnormal values, compared to the threshold are detected, in both the vehicular and biomedical sensors, then an accident is confirmed by the processor. Information about the accident is them sent to the alert module by the local processor. Also, the local processor obtains information regarding the location of the on-board
GPS. This ensures its ability to continuously locate the vehicle.

(2) Speed monitor module: it is used to maintain a record of the speed at which the vehicle is travelling and records the average speed at which the vehicle travels at each road. This message is then transmitted to the message and alert module.

(3) Message and alert module: it is used to maintain communication with the central server, RSU units, 
and the ambulance. It also obtains the average speed, from the speed monitor module, at which the vehicle is travelling at each section of the road. This information is then transmitted to the corresponding RSU unit.

3.2.2. Central Server Unit. The central server unit is central to our model which has connections to all the entity units. It is the responsibility of the central server to manage traffic. It contains two modules:

(1) Speed map module: it uses the average speeds that vehicles are travelling at to create a roadmap of each road.

(2) Optimal path module: it is used to compute the best path that an ambulance can take to the scene of the accident and also to the hospital.

3.2.3. RSU Unit. The RSU unit has the responsibility of monitoring and managing traffic and also consists of two modules.

(1) Vehicle monitor module: it is used to collect the average speeds at which vehicles are travelling in the adjacent section of the road. This information is then transmitted to the central server unit.

(2) Ambulance monitor module: it is used for tracking the ambulance and setting traffic lights along the route in a manner that reduces the time an ambulance has to wait. Our model divides roads into road segments between two RSUs. A distinct road ID is provided for each road segment. Every RSU, at the beginning of a road segment, transmits the road ID through which the vehicle is going to pass. Once each vehicle gets the message, it begins to monitor its speed on that segment of the road. Once the vehicle arrives at the end of the road segment, it transmits a message to the RSUs. Within each message, there are two fields: the matching road ID and average speed. The RSUs then gather the vehicle's speed details from the vehicle and then compute the aggregate average speeds of all vehicles, which are then transmitted by the RSUs to the central server. This is the information that the central server then uses to create the city's speed roadmap.

3.2.4. Ambulance Unit. The vehicular unit and the central server unit provide the ambulance with accident information and the optimal route information, respectively. With the assistance of the GPS, the ambulance then uses this information to identify the best route. As it travels, the ambulance will be transmitting an alert message which contains two fields: the current and next road IDs. At the beginning of each road segment, upon the RSU receiving the alert message containing the current road ID, it transmits the message to the RSU at the end of the road segment. Once the transmitted message has been received, the RSU will extract the following segment of the road that the ambulance is travelling towards and sets the traffic light to allow the ambulance quick passage. This will be done all the way until the ambulance arrives at the place where it is going.

3.2.5. Hospital Unit. There is a connection between the hospital unit, the ambulance units, and the central server. Health updates about the situation of the patient are continuously transmitted to the hospital unit, directly from the ambulance. This ensures that the treatment is started as soon as possible once the patient arrives at the hospital. Owing to the fact that the ambulance keeps the hospital informed of its current location, the hospital is able to obtain the patient's preliminary health status ahead of the ambulance arriving at the hospital. Since the location of the ambulance is updated, the hospital is able to estimate the time at which the patient will arrive at the hospital and ensure preliminary steps for the patient's treatment.

\section{Proposed Traffic Routing Algorithms}

In this section, we discuss the postaccident action in our system. Traffic routing consists of two proposed algorithms. First, we introduce the multihop optimal forwarding algorithm which provides the optimal route between accident vehicle and ambulance. Second, we introduce the optimal route planning algorithm which provides the optimal route algorithm from ambulance to accident vehicle and then to hospital.

4.1. Multihop Optimal Forwarding Algorithm. This section provides the details of how we plan to use the sources (accident vehicle) to identify the optimal route to the destination (ambulance) on a hop-by-hop basis. The algorithm is based on a V2V communications. Figure 3 shows the green vehicle as the source and Ambulance 1 is shown as destination 1 (D1), which is located at I3, while Ambulance 2 is destination 2 (D2) whose location is I4.

In case of an accident, the source sends a packet to the ambulance nearest to it. The accident vehicle computes the length of the path from all intersections that link it with both destinations and chooses the destination whose path length (PL) is the shortest. With regard to D1, from Figure 3, using the red arrows PL2D1 < PL5D1, it can be shown that the packet forwarded to D1 from intersection I2-I3 will be faster than the one to D1 from I5-I6-I3. Accordingly, the accident will require two intersections to get to Ambulance 1. Meanwhile, with regard to D2, as shown in Figure 3 by green arrows, PL5D2 < PL2D2, the packet to intersection I5I4 to D2 will be quicker when compared to the packet transmitted from I2-I1-I4. Based on this, the accident vehicle will choose the path that has more vehicles to ensure continuous connectivity. Consequently, two intersections are required by the accident vehicle in order for it to reach Ambulance 2. What this implies is that we have two paths whose length is similar: I5-I4-D2 and I2-13-D1. For this case, it would be more prudent for the accident vehicle to choose a path that has more vehicles so that maximum connectivity can be maintained. According to Figure 3, the path chosen by the accident vehicle is PL2. 


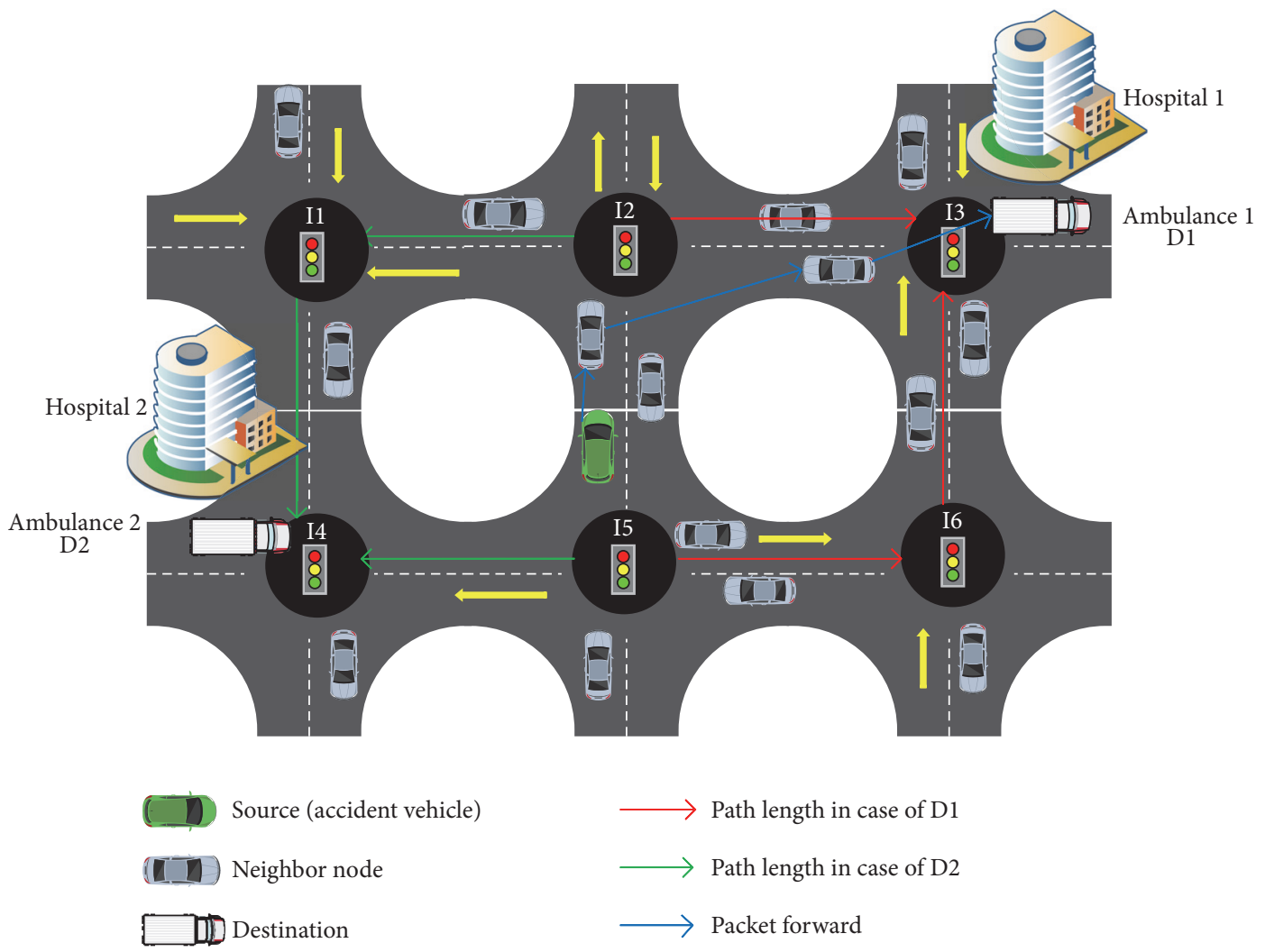

FIgURE 3: Source vehicle calculates the path length to destination.

The blue arrows denote the accident vehicle forwarding the packet to the next candidate vehicle travelling towards the intersection that has the shortest path length to D1, depending on $[35,36]$. This is a process that continues all the way until the packet arrives at its estimation. This not only does ensure fast progress, but also makes sure that there is minimal traffic on the network.

4.2. Optimal Route Planning Algorithm. Our aim in this section is to identify the optimal route planning algorithm (ORPA) from the ambulance to the accident vehicle and then to the hospital. The reasoning behind this algorithm is the avoidance of traffic congestion and the reduction of the average cost of travelling.

It is expected that each vehicle will trial a planned route right from the point where it starts its journey to the point where the journey terminates. In this instance, the planned route will be referred to, in GPS, as a route preset, depending on what the choices of the driver are and also depending on where the starting and termination points are located. The driver is also expected to follow the route which has been preset until they receive an alert that there is congestion along that route. The central server, through managing the optimal route planning algorithm, will be responsible for identifying the optimal substitute routing for a particular ambulance once congestion or an accident happens. To facilitate the understanding of technical aspects, a table of notations would be useful as illustrated in Table 1.
The optimal route planning algorithm is planned in a manner that balances the avoidance of congestion while also ensuring that the cost of travel is reduced. Hence, for this purpose, we suggest two metrics. The first one is the weighted average cost metric which determines the average cost for vehicles whose routes have been replanned, while also ensuring that the preferences of the driver are also considered. Some of the mathematical notions used in this algorithm are $v, d,(i, j)$, and $T$ representing vehicles, destination, road segment, and sample time duration, respectively. In this case, we consider $\mathrm{PL}_{c i}^{v d}$ as the prevailing length of the path (first based on what the driver prefers such as the one they are familiar with or the shortest one), where $c i$ is the path selected ahead of the replanning. $\mathrm{PL}_{p i j}^{v d}$ represents the changed path, where, pij represents the new path which has been planned and by going via $(i, j)$ the path of $v$ is changed in the direction of $d . C(T)$ is vehicle travel cost and will be formulated as

$$
\begin{aligned}
& C(T) \\
& \quad= \begin{cases}0, & \text { if } i \notin \text { current path of } v d \\
\rho\left(\left|\mathrm{PL}_{p i j}^{v d}\right|-\left|\mathrm{PL}_{c i}^{v d}\right|\right), & \text { if } i \in \text { current path of } v d\end{cases}
\end{aligned}
$$

where $\rho$ represents a nonnegative accumulative function used to measure the effect of the increase in path length [37]. 
TABLE 1: Summary of the important mathematical notations.

\begin{tabular}{|c|c|}
\hline Symbol & Description \\
\hline $\mathbf{v}$ & Vehicles \\
\hline d & Destination \\
\hline$(i, j)$ & Road segment \\
\hline$T$ & Sample time duration \\
\hline$P L_{c i}^{v d}$ & Prevailing length of the path \\
\hline$c i$ & The path selected ahead of the re-planning \\
\hline $\mathbf{P L}_{\mathrm{pij}}^{\mathrm{vd}}$ & The changed path \\
\hline pij & The new path \\
\hline $\mathbf{C}(\mathbf{T})$ & Vehicle travel cost \\
\hline$\rho$ & A nonnegative accumulative function \\
\hline $\mathrm{C}_{\mathrm{ij}}(\mathrm{T})$ & The average cost of vehicles on road segment \\
\hline $\mathrm{C}_{\mathbf{i}}(\mathrm{T})$ & The average cost of vehicles on intersection $i$ \\
\hline $\mathbf{J}_{\mathbf{i}}$ & The set of adjacent crossing of intersection $i$ \\
\hline$\lambda_{\mathrm{ij}}(\mathrm{T})$ & The average inflow rates of road segment \\
\hline$\mu_{\mathrm{ij}}(\mathrm{T})$ & The average outflow rates of road segment \\
\hline$\lambda_{\mathrm{ij}}(\mathbf{t})$ & The upstream vehicle arrivals respectively on road segment \\
\hline$\mu_{\mathrm{ij}}(\mathrm{t})$ & The upstream vehicle departure rates on road segment \\
\hline$\Delta$ & Sample time duration \\
\hline $\mathbf{Q}_{\mathbf{i}}(\mathbf{T})$ & The total length of all virtual queues of intersection $i$ \\
\hline WI & Weighted intersection \\
\hline $\mathbf{W I}(\mathbf{T})$ & Intersection $i$ 's weight in sample time \\
\hline $\mathbf{Q}_{\mathbf{i}}^{\mathbf{d}}(\mathbf{T})$ & Virtual queues representing the number of the shielded vehicles travelling through intersection $i$ \\
\hline$Q_{j}^{\mathbf{d}}(T)$ & Virtual queues representing the number of the shielded vehicles travelling through intersection $j$ \\
\hline $\mathbf{j} *$ & The huge differential row build-up for intersection $i$ and the other adjacent intersection $j$ \\
\hline $\mathbf{q}_{\mathbf{j} *}^{\mathrm{d}}(\mathbf{T})$ & Re-planning to intersection $j *$ of a number of vehicles \\
\hline
\end{tabular}

Computing the average cost of vehicles on road segment $(i, j)$ can be done as follows:

$$
C_{i j}(T)=\frac{\sum C(T)}{V}
$$

where $V$ represents the number of vehicles travelling on a road segment $(i, j)$. For an intersection, considering that there could be a number of adjacent intersections which are candidates of the approaching intersections, determining the average cost of vehicles on intersection $i$ is defined as

$$
C_{i}(T)=\frac{\sum_{j \in J_{i}} C_{i j}(T)}{J_{i}}
$$

where $J_{i}$ represents the set of adjacent crossing of intersection $i$. Secondly, in the weighted network stability, we used the inflow/outflow system (Figure 4) as a method of modelling vehicle traffic [38].

With the Tth time interval based on the rates of the traffic flow involved, as gathered by RSUs, the average inflow and outflow rates of road segment $(i, j)$ are marked as $\lambda_{i j}(T)$ and $\mu_{i j}(T)$, respectively, and formulated as

$$
\lambda_{i j}(T)=\frac{1}{\Delta} \sum_{t=(T-1) \Delta}^{T \Delta} \lambda_{i j}(t)
$$

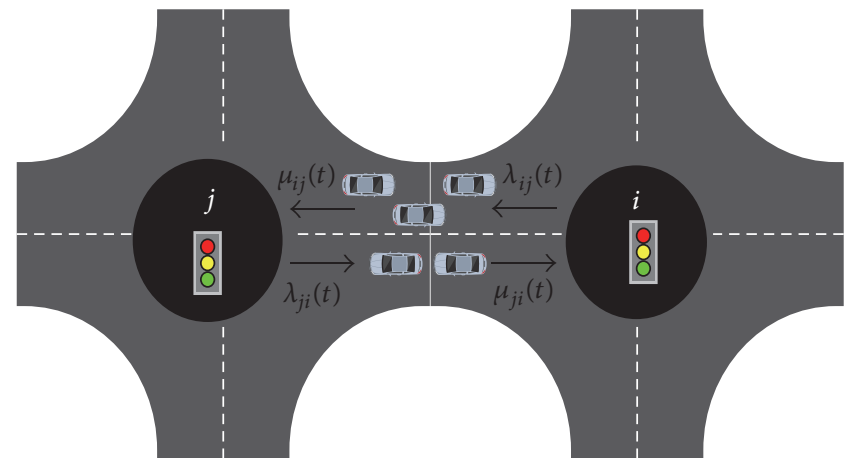

Figure 4: Traffic flow model.

$$
\mu_{i j}(T)=\frac{1}{\Delta} \sum_{t=(T-1) \Delta}^{T \Delta} \mu_{i j}(t)
$$

where $\lambda_{i j}(t)$ and $\mu_{i j}(t)$ denote the upstream vehicle arrivals and departure rates, respectively, on road segment $(i, j)$ whose destination on the timeslot $t$ is the same. $\Delta$ is a sample time duration that includes a series of time slots as distinguished by sampling theorem with the aim of mitigating the risk of loss of information in the compressive sensing for 
estimation of traffic in [39]. It is vital to be conscious of the reality that all variables for the road segment $(i, j)$ on the opposite direction identified as road segment $(j, i)$ could be defined as $\lambda_{j i}(T)$ and $\mu_{j i}(T)$, respectively. The information on a virtual queue of length $Q_{i}(T)$ is needed in order to determine if an intersection is stable. Defining the leftover number of vehicles on road segment $(i, j)$ will be done as follows:

$$
Q_{i j}(T)=\lambda_{i j}(T-1)+\mu_{i j}(T-1)
$$

From (5) we can get the total length $Q_{i}(T)$ of all virtual queues of intersection $i$ for all destinations as

$$
Q_{i}(T)=\sum_{j \in J i} Q_{i j}(T)
$$

Provided that there is stability on all queues within the network, the traffic flow across the whole network will equally be stable. In the event that the central server gets an incident warning message gathered by vehicles through VANETs and RSUs, the optimal route planning algorithm will be computed to update and also determine $\lambda_{i j}(T)$ with the aim of reducing the cost of travelling and improving spatial utility. With regard to the time on the sample, $T$, based on the optimal route planning algorithm, it is possible to dispatch a vehicle whose destination is $d$ from one intersection towards another. The required vehicle will be navigated through the updated path as delivered to the GPS device. To meet the goal of balancing between reduce travel cost and spatial utility, we have designed an innovative metric referred to as weighted intersection (WI). Allow WI(T) to represent intersection $i$ 's weight in sample time $T$, formulated as shown below:

$$
\mathrm{WI}_{i}(T)=\left[Q_{i}^{d}(T)-Q_{j}^{d}(T)\right]-K C_{i}(T)
$$

where $Q_{i}^{d}(T)$ and $Q_{j}^{d}(T)$ are virtual queues representing the number of the shielded vehicles travelling through intersections $i$ and $j$ in the direction of destination $d$ in sample time $T . K$ is a nonnegative constant defined by the central server used for every vehicle [40]. In the beginning, vehicles travelling through an intersection that has a heavy weight are replanned. Eventually, vehicles with destination $d$, kept at $i$, have to be dispatched to queue $Q_{j *}^{d}(T)$ of intersection $j *$, where $j *$ represents the huge differential row build-up for intersection $i$ and the other adjacent intersection $j$ and destination $d$ as

$$
j *=Q_{i}^{d}(T)-Q_{j}^{d}(T)
$$

According to short increased path lengths, there will be a replanning to intersection $j *$ of a number of vehicles whose destination is $d$, using

$$
q_{j *}^{d}(T)=Q_{i}^{d}(T)-Q_{j *}^{d}(T)
$$

Subsequently, queues at all remaining intersections are correspondingly updated as follows:

$$
\begin{gathered}
Q_{i}^{d}(T)=Q_{i}^{d}(T)-q_{j *}^{d}(T) \\
Q_{j *}^{d}(T)=Q_{j *}^{d}(T)+q_{j *}^{d}(T)
\end{gathered}
$$

A similar process continues all the way till all linked intersections have been processed. Algorithm 1 shows the detail of OPPA.

\section{Performance Evaluation}

For purposes of conducting the case study, a general Manhattan grid model has been selected. In order to design the scenario and extract the intersection images, MATLAB and Google Maps are used, respectively. Simulations that deploy assistance mobility models with the capacity to make vehicles accelerate, change direction, and decelerate, can enable realistic scenarios to be achieved. For this part of the study, a familiar mobility model which is car following model [4] is used. Using the average speed information obtained from the RSUs, the map road segment is separated into two speed zones. If the average vehicle speed on a certain road segment is low, then that segment is considered congested and those with high average speeds are seen as free from congestion. However, the method of incessantly averaging speeds of vehicles is prone to errors as a result of accumulated historical data. The result of this is that the data represents a historical characteristic as opposed to the prevailing average speed at a given time. To mitigate against this challenge, we create divisions based on 5-minute time slots with the aggregate average being computed in any slot using the data obtained from only that slot. Another challenge is the high overhead resulting from regular updates of speed between central servers and RSUs. To mitigate this problem, the speed will be separated into time zones, with the RSU only updating the average speed if there are any changes in the average speed from one time zone to the next. Based on the RSU, the central server update will dynamically provide updates of the time zone to reflect the road segment's congestion.

We do a comparison of the assessment of four algorithms within our ambulance setting using their average speed and travelling time as a basis. The Dijkstra algorithm deploys the characteristic for planning paths without any changes. In this case, each road segment speed assigns a distinct average value for all segments. When using this algorithm, congestion is not considered, and speed cannot be dynamically altered. There are similarities between that the $A *$ algorithm and the Dijkstra. However, in the A* algorithm the experimental function $H(n)$ is presented. The $H(n)$ denotes the angle between the adjacent node and the straight line in the direction of the endpoint node. With regard to the $\mathrm{VBA} *$ algorithm, the prevailing speed on a road segment can be varied, dynamically, and a distribution manner can be used to operate it. We finally do a comparison of the proposed ORPA, A*, Dijkstra, and VBA* algorithms. The implemented simulation parameters are presented in Table 2 .

Figure 5 shows the travelling time of Dijkstra, $\mathrm{A} *, \mathrm{VBA} *$, and ORPA in the low- and high-density scenario. The ORPA obtains a lower travelling time than Dijkstra, A* and VBA*. Specifically, in low-density the travelling time of ORPA, Dijkstra, A*, and VBA* reaches 1400, 2400, 2200, and 2000 seconds, respectively. Hence, the travelling time of ORPA is $71.4 \%, 57.14 \%$, and $42.85 \%$ lower than that of Dijkstra, $\mathrm{A} *$, and $\mathrm{VBA} *$, respectively, whereas in high-density the ORPA 


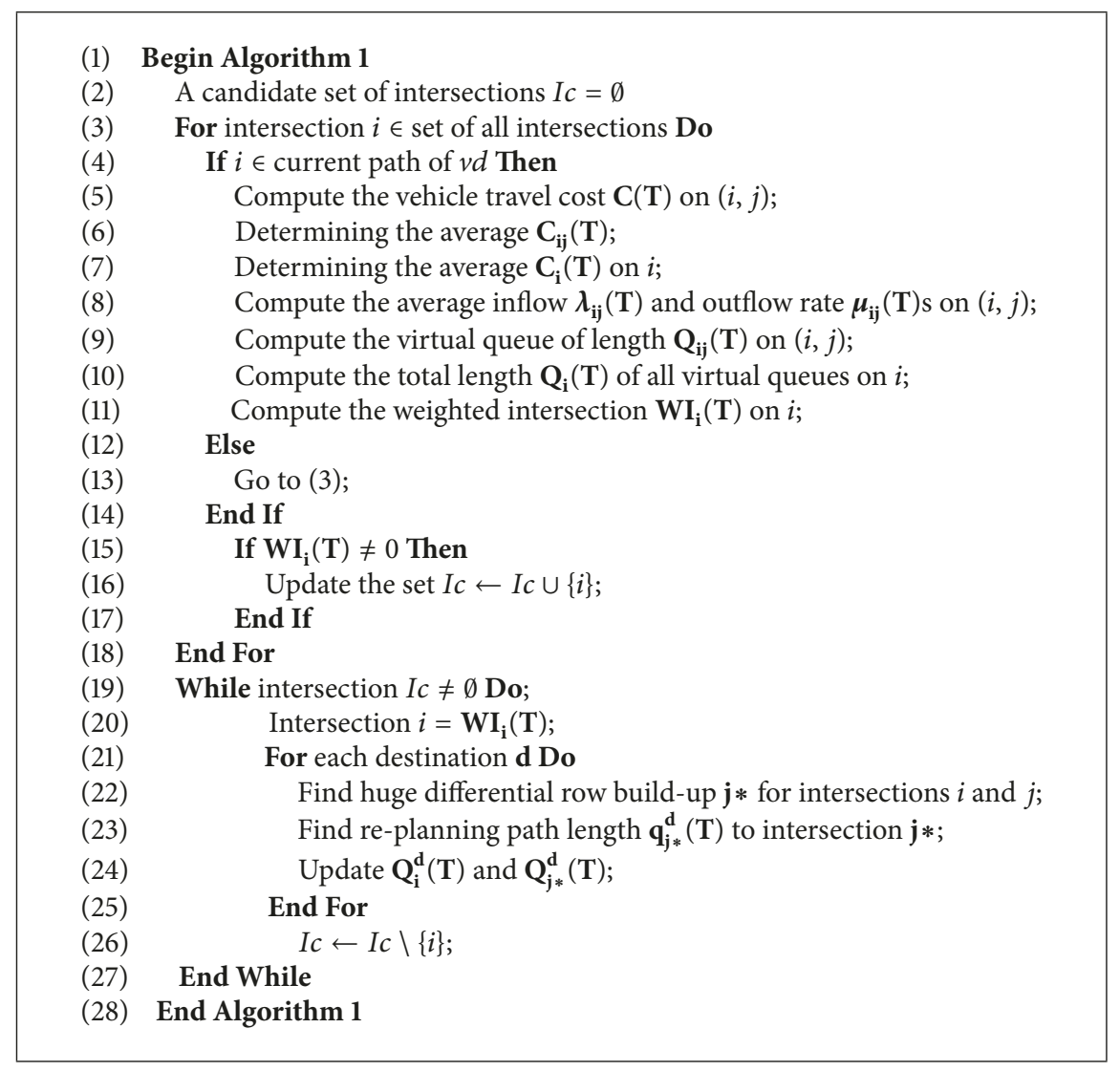

Algorithm 1: ORPA.

TABLE 2: Simulation parameters.

\begin{tabular}{lc}
\hline Parameter & Value \\
\hline Simulation area & $5 \times 5 \mathrm{~km}^{2}$ \\
\hline Number of lanes & 2 bidirectional \\
\hline Intersections & 24 \\
\hline Segments & 38 \\
\hline Speed & $(40-80) \mathrm{km} / \mathrm{hr}$. \\
\hline & $\begin{array}{l}\text { High density } \\
\text { Average vehicles density }\end{array}$ \\
\hline Communication radius & density $6-8$ veh $/ \mathrm{lane} / \mathrm{km}$ \\
\hline MAC protocol & $300 \mathrm{~m}$ \\
\hline Total simulation time & IEEE $802.1 \mathrm{p}$ \\
\hline
\end{tabular}

obtains a travelling time of 2100 seconds, while Dijkstra, $\mathrm{A} *$, and VBA obtain a travelling time of 3200,3000 , and 2700 seconds. Thus, ORPA decreases the total travelling time by $52.38 \%, 42.3 \%$, and $28.57 \%$ for Dijkstra, $\mathrm{A} *$, and VBA, respectively. Figure 5 illustrates that the travelling time of high-density is greater than low-density due to congestion.

Figure 6 depicts the average ambulance speed of four algorithms in low- and high-density scenario. The ORPA obtains a higher average ambulance speed than Dijkstra, $A *$, and $V B A *$. Specifically, in low-density the average ambulance

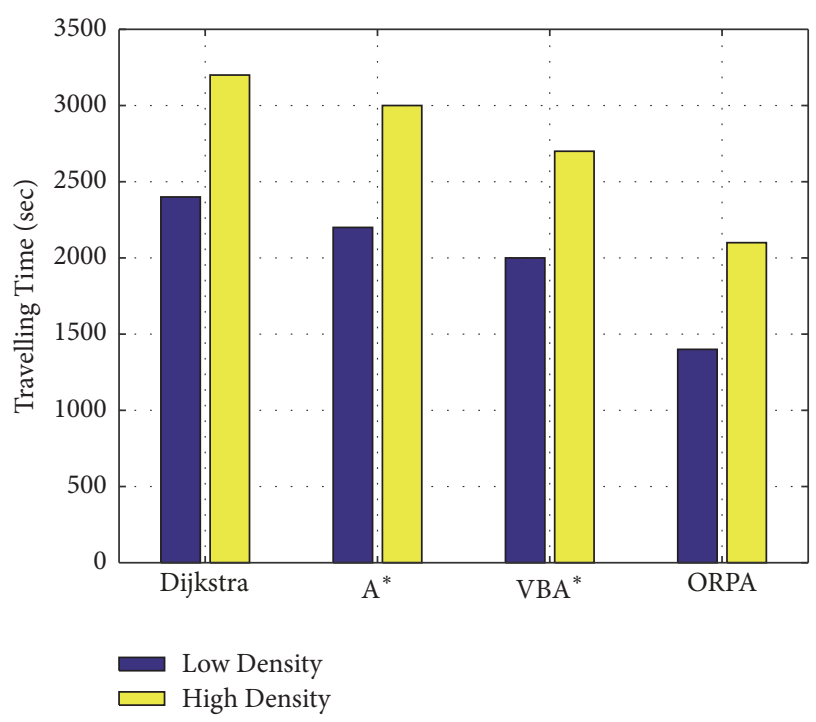

FIGURE 5: Travelling time in low- and high-density.

speed of ORPA, Dijkstra, A*, and VBA* reaches $45,23,26$, and $32 \mathrm{~km} / \mathrm{hr}$, respectively. Hence, the average ambulance speed of ORPA is $48.8 \%, 42.2 \%$, and $28.8 \%$ higher than that of Dijkstra, $\mathrm{A} *$, and $\mathrm{VBA} *$, respectively, whereas in highdensity the average ambulance speed of ORPA, Dijkstra, A*, and $\mathrm{VBA} *$ reaches $30,12,17$, and $20 \mathrm{~km} / \mathrm{hr}$, respectively. 


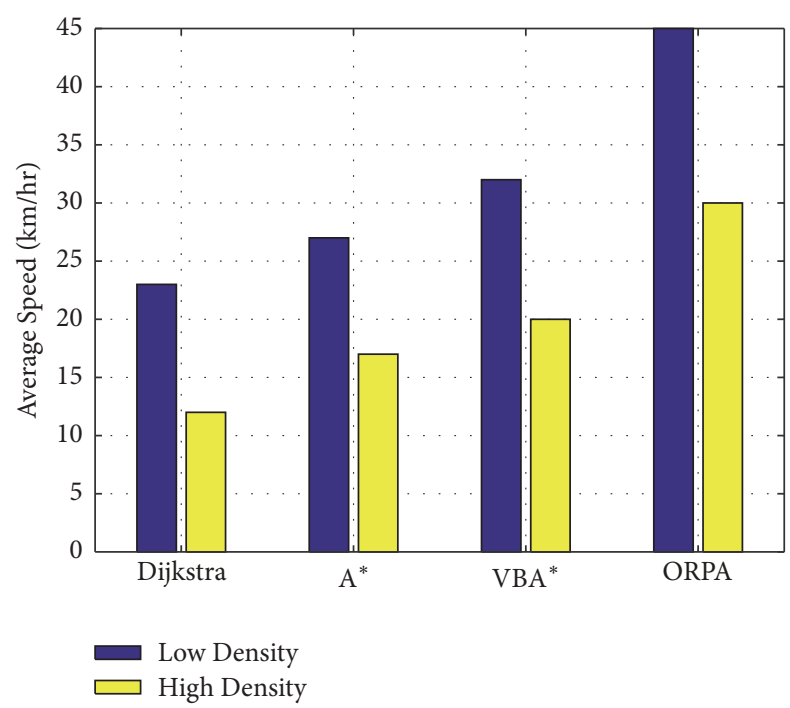

FIgURE 6: Average ambulance speed in low- and high-density.

Thus, the average ambulance speed of ORPA is $60 \%, 43.3 \%$, and $33.3 \%$ higher than that of Dijkstra, A*, and VBA*, respectively. This figure further depicts that the average ambulance speed of low-density environments is greater than that of high-density environments.

\section{Conclusion}

Through this paper, we have developed a common system to manage accidents so that vehicles are able to avoid congested areas within an ITS. Initially, we established an accident management system which employs cellular systems of the public transportation systems and VANETs to make efficient real-time communication between vehicles possible, including ambulances, hospitals, RSUs, and central servers. We subsequently propose a real-time algorithm for planning routes with the aim of improving the overall use of space while at the same time reducing the cost of travelling, through vehicles' ability to avoid congested road segments. Finally, we have shown that the path planning algorithm we propose will reduce the time taken by ambulances to be alerted and dispatched to a scene of accident through being able to avoid road segments that are congested and will increase the chance of saving the lives of accident victims.

\section{Data Availability}

There is no data set related to our work.

\section{Conflicts of Interest}

The authors declare that there are no conflicts of interest regarding the publication of this paper.

\section{Acknowledgments}

This work is fully funded by Universiti Kebangsaan Malaysia under Grant no. GUP-2016-005.

\section{References}

[1] M. Y. I. Idris, A. M. A. Abu Znaid, A. W. A. Wahab, L. K. Qabajeh, and O. A. Mahdi, "Low communication cost (LCC) scheme for localizing mobile wireless sensor networks," Wireless Networks, vol. 23, no. 3, pp. 737-747, 2017.

[2] A. S. Sadiq, S. Khan, K. Z. Ghafoor, M. Guizani, and S. Mirjalili, "Transmission power adaption scheme for improving IoV awareness exploiting: evaluation weighted matrix based on piggybacked information," Computer Networks, vol.137, pp. 147$159,2018$.

[3] G. Egilmez and D. McAvoy, "Benchmarking road safety of U.S. states: A DEA-based Malmquist productivity index approach," Accident Analysis \& Prevention, vol. 53, pp. 55-64, 2013.

[4] H. Hartenstein and K. Laberteaux, VANET: Vehicular Applications and Inter-Networking Technologies, vol. 1, John Wiley \& Sons, 2009.

[5] Y. R. B. Al-Mayouf, N. F. Abdullah, M. Ismail, A. W. A. Wahab, and O. A. Mahdi, "Efficient routing algorithm for VANETs based on distance factor," in Proceedings of the 2016 International Conference on Advances in Electrical, Electronic and Systems Engineering, ICAEES 2016, pp. 567-571, mys, November 2016.

[6] Y. Sun, R. Lu, X. Lin, X. Shen, and J. Su, "An efficient pseudonymous authentication scheme with strong privacy preservation for vehicular communications," IEEE Transactions on Vehicular Technology, vol. 59, no. 7, pp. 3589-3603, 2010.

[7] Y. R. B. Al-Mayouf, M. Ismail, N. F. Abdullah, S. M. AlQaraawi, and O. A. Mahdi, "Survey on VANET technologies and simulation models," ARPN Journal of Engineering and Applied Sciences, vol. 11, no. 15, pp. 9414-9427, 2016.

[8] M. Wang, H. Liang, R. Zhang, R. Deng, and X. Shen, "Mobilityaware coordinated charging for electric vehicles in VANETenhanced smart grid," IEEE Journal on Selected Areas in Communications, vol. 32, no. 7, pp. 1344-1360, 2014.

[9] I. Leontiadis, G. Marfia, D. Mack, G. Pau, C. Mascolo, and M. Gerla, "On the effectiveness of an opportunistic traffic management system for vehicular networks," IEEE Transactions on Intelligent Transportation Systems, vol. 12, no. 4, pp. 15371548, 2011.

[10] O. A. Mahdi, A. W. Abdul Wahab, M. Y. I. Idris et al., "A comparison study on node clustering techniques used in target tracking WSNs for efficient data aggregation," Wireless Communications and Mobile Computing, vol. 16, no. 16, pp. 2663-2676, 2016.

[11] A. Skabardonis and N. Geroliminis, "Real-time monitoring and control on signalized arterials," Journal of Intelligent Transportation Systems: Technology, Planning, and Operations, vol. 12, no. 2, pp. 64-74, 2008.

[12] N. M. Drawil and O. Basir, "Intervehicle-communicationassisted localization," IEEE Transactions on Intelligent Transportation Systems, vol. 11, no. 3, pp. 678-691, 2010.

[13] O. A. Mahdi, A. W. Abdul Wahab, M. Y. I. Idris, A. Abu Znaid, Y. R. B. Al-Mayouf, and S. Khan, "WDARS: a weighted data aggregation routing strategy with minimum link cost in eventdriven WSNs," Journal of Sensors, vol. 2016, Article ID 3428730, 12 pages, 2016.

[14] A. M. A. Abu Znaid, M. Y. I. Idris, A. W. Abdul Wahab, L. Khamis Qabajeh, and O. Adil Mahdi, "Sequential Monte Carlo Localization Methods in Mobile Wireless Sensor Networks: A Review," Journal of Sensors, vol. 2017, Article ID 1430145, 2017.

[15] A. H. Khosroshahi, P. Keshavarzi, Z. D. Koozehkanani, and J. Sobhi, "Acquiring real time traffic information using VANET 
and dynamic route guidance," in Proceedings of the IEEE 2nd International Conference on Computing, Control and Industrial Engineering, CCIE 2011, pp. 9-13, August 2011.

[16] O. A. Mahdi, A. W. Abdul Wahab, M. Y. I. Idris, A. Abu Znaid, S. Khan, and Y. R. B. Al-Mayouf, "ESAM: endocrine inspired sensor activation mechanism for multi-target tracking in WSNs", in Proceedings of the 4th International Conference on Wireless and Optical Communications, vol. 9902 of Proceedings of SPIE, International Society for Optics and Photonics, Beijing, China, June 2016.

[17] M. Abboud, L. Jaoude, and Z. Kerbage, "Real time GPS navigation system," in Proceedings of the 3rd FEA Student Conference, Department of Electrical and Computer Engineering, American University of Beirut, Citeseer, 2004.

[18] Y. Chung, "Development of an accident duration prediction model on the Korean Freeway Systems," Accident Analysis \& Prevention, vol. 42, no. 1, pp. 282-289, 2010.

[19] Y. Zhao, K. Triantis, D. Teodorović, and P. Edara, "A travel demand management strategy: The downtown space reservation system," European Journal of Operational Research, vol. 205, no. 3, pp. 584-594, 2010.

[20] J. C. Herrera, D. B. Work, R. Herring, X. Ban, Q. Jacobson, and A. M. Bayen, "Evaluation of traffic data obtained via GPSenabled mobile phones: the Mobile Century field experiment," Transportation Research, Part C: Emerging Technologies, vol. 18, no. 4, pp. 568-583, 2010.

[21] R. Herring et al., "Using mobile phones to forecast arterial traffic through statistical learning," in Proceedings of the 89th Transportation Research Board Annual Meeting, 2010.

[22] J. Jariyasunant et al., Mobile transit trip planning with real-time data, 2011, Mobile transit trip planning with real-time data.

[23] B. Hoh, M. Gruteser, R. Herring et al., "Virtual trip lines for distributed privacy-preserving traffic monitoring," in Proceedings of the 6th international conference on Mobile systems, applications, and services, p. 15, Breckenridge, CO, USA, June 2008.

[24] H. T. Cheng, H. Shan, and W. Zhuang, "Infotainment and road safety service support in vehicular networking: from a communication perspective," Mechanical Systems and Signal Processing, vol. 25, no. 6, pp. 2020-2038, 2011.

[25] J. Zhao and G. Cao, "VADD: vehicle-assisted data delivery in vehicular Ad hoc networks," IEEE Transactions on Vehicular Technology, vol. 57, no. 3, pp. 1910-1922, 2008.

[26] T. H. Luan, X. Ling, and X. Shen, "Provisioning QoS controlled media access in vehicular to infrastructure communications," Ad Hoc Networks, vol. 100, no. 2, pp. 231-242, 2012.

[27] C. Xu, F. Zhao, J. Guan, H. Zhang, and G.-M. Muntean, "QoEdriven user-centric vod services in urban multihomed P2Pbased vehicular networks," IEEE Transactions on Vehicular Technology, vol. 62, no. 5, pp. 2273-2289, 2013.

[28] L. Zhou, Y. Zhang, K. Song, W. Jing, and A. V. Vasilakos, "Distributed media services in P2P-based vehicular networks," IEEE Transactions on Vehicular Technology, vol. 60, no. 2, pp. 692-703, 2011.

[29] P. Chen, Y. Guo, and W. Chen, "Fuel-Saving Navigation System in VANETs," in Proceedings of the 2010 IEEE Vehicular Technology Conference (VTC 2010-Fall), pp. 1-5, Ottawa, ON, Canada, September 2010.

[30] M. Kimura, Y. Taoda, Y. Kakuda, S. Inoue, and T. Dohi, "A novel method based on VANET for alleviating traffic congestion in urban transportations," in Proceedings of the 2013 IEEE Eleventh
International Symposium on Autonomous Decentralized Systems (ISADS), pp. 1-7, Mexico City, Mexico, March 2013.

[31] T. Schouwenaars, B. De Moor, E. Feron, and J. How, "Mixed integer programming for multi-vehicle path planning," in Proceedings of the 6th European Control Conference, ECC 2001, pp. 2603-2608, prt, September 2001.

[32] H. Saleet, O. Basir, R. Langar, and R. Boutaba, "Regionbased location-service-management protocol for VANETs," IEEE Transactions on Vehicular Technology, vol. 59, no. 2, pp. 917-931, 2010.

[33] F. Li and Y. Wang, "Routing in vehicular ad hoc networks: a survey," IEEE Vehicular Technology Magazine, vol. 2, no. 2, pp. $12-22,2007$.

[34] R. N. Murty, G. Mainland, I. Rose et al., "Citysense: an urbanscale wireless sensor network and testbed," in Proceedings of the IEEE Conference on Technologies for Homeland Security, pp. 583-588, IEEE, 2008.

[35] Y. R. B. Al-Mayouf, N. F. Abdullah, M. Ismail, S. M. Al-Qaraawi, O. A. Mahdi, and S. Khan, "Evaluation of efficient vehicular ad hoc networks based on a maximum distance routing algorithm," EURASIP Journal on Wireless Communications and Networking, vol. 2016, no. 1, article no. 265, 2016.

[36] Y. R. Al-Mayouf, M. Ismail, N. F. Abdullah et al., "Efficient and stable routing algorithm based on user mobility and node density in urban vehicular network," PLoS ONE, vol. 11, no. 11, Article ID e0165966, 2016.

[37] H. Liang, B. J. Choi, W. Zhuang, and X. Shen, "Optimizing the energy delivery via V2G systems based on stochastic inventory theory," IEEE Transactions on Smart Grid, vol. 4, no. 4, pp. 2230 2243, 2013.

[38] J. Chen, W. Xu, S. He, Y. Sun, P. Thulasiraman, and X. Shen, "Utility-based asynchronous flow control algorithm for wireless sensor networks," IEEE Journal on Selected Areas in Communications, vol. 28, no. 7, pp. 1116-1126, 2010.

[39] Z. Li, Y. Zhu, H. Zhu, and M. Li, "Compressive sensing approach to urban traffic sensing," in Proceedings of the 31st International Conference on Distributed Computing Systems (ICDCS '11), pp. 889-898, IEEE, July 2011.

[40] M. J. Neely, "Stochastic network optimization with application to communication and queueing systems," Synthesis Lectures on Communication Networks, vol. 3, no. 1, 211 pages, 2010. 


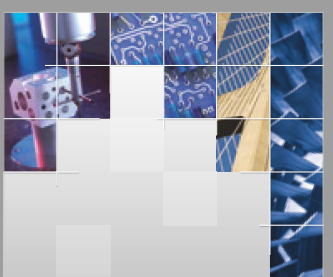

\section{Enfincering}
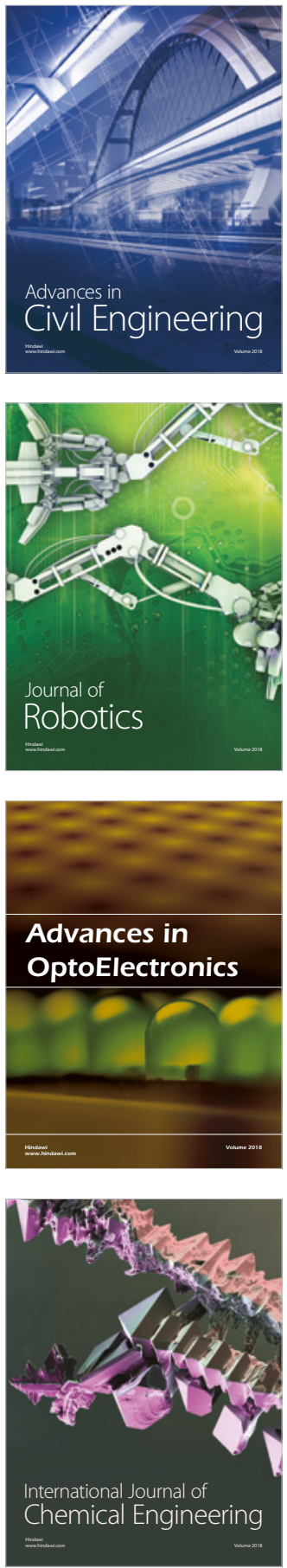

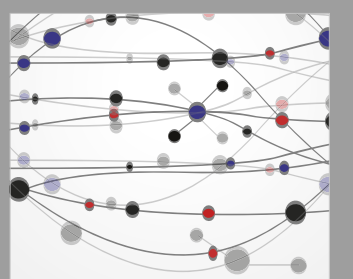

\section{Rotating \\ Machinery}

The Scientific World Journal

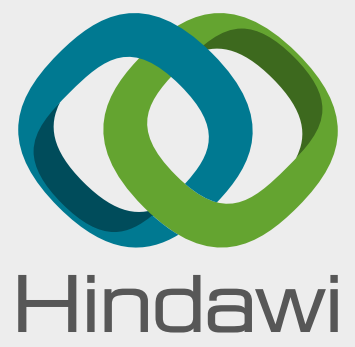

Submit your manuscripts at

www.hindawi.com
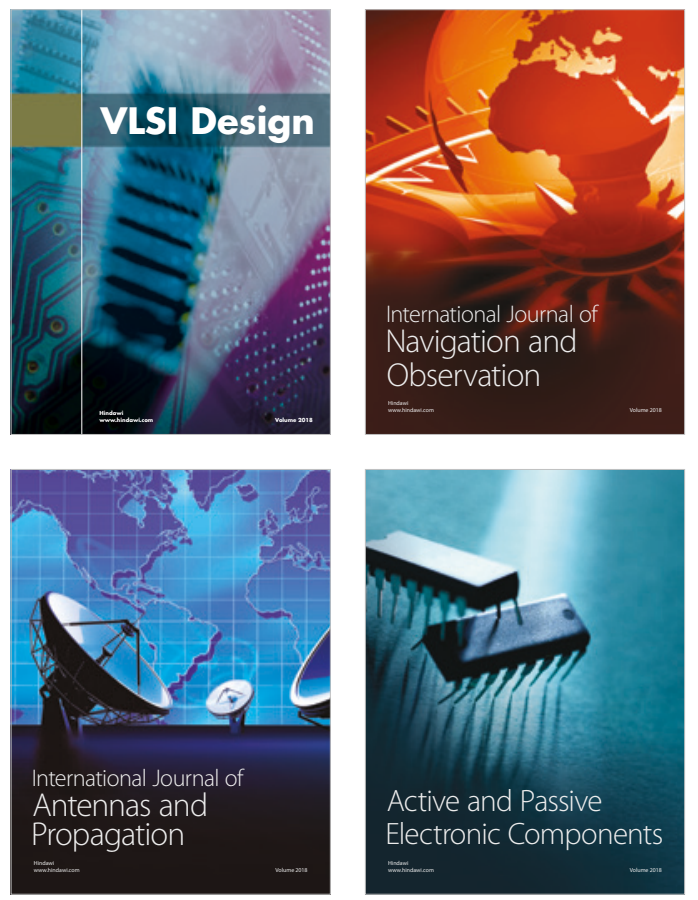
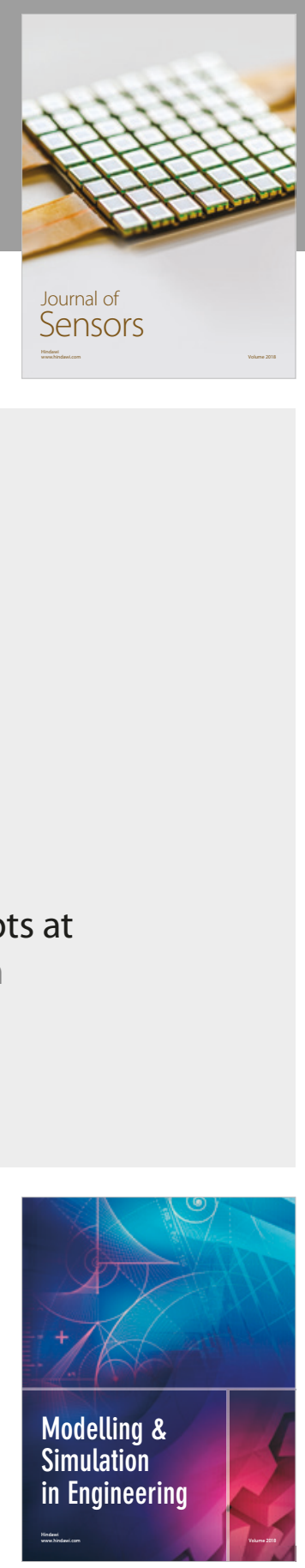

\section{Advances \\ Multimedia}
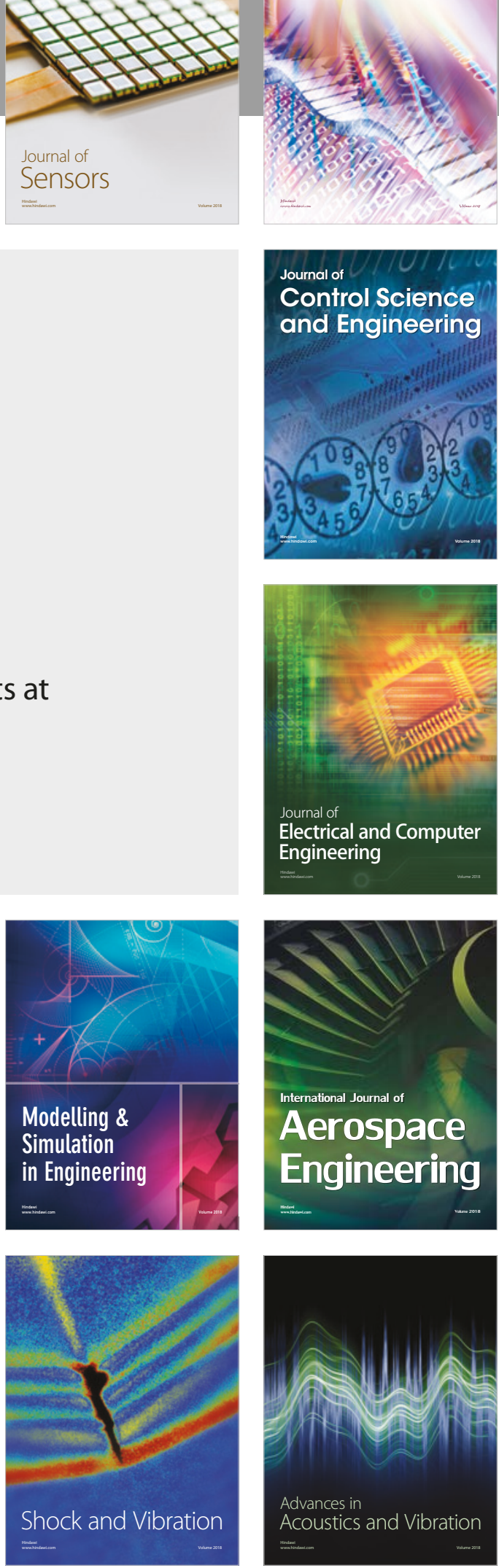